\title{
NOVEL CdTe/CdMgTe GRADED QUANTUM WELL STRUCTURES*
}

\author{
T. WoJtowicz, M. Kutrowski, G. KarczewsKi, G. CYWIŃski, \\ M. Surma, J. Kossut \\ Institute of Physics, Polish Academy of Sciences \\ Al. Lotników 32/46, 02-668 Warsaw, Poland \\ D.R. Yakovlev, W. Ossau, G. Landwehr \\ Physikalisches Institut der Universität Würzburg \\ Am Hubland, 97074 Würzburg, Germany \\ AND V. KOCHERESHKO
}

A.F. Ioffe Physico-Technical Institute, Russian Academy of Sciences

St. Petersburg, Russia

We report on growth and magnetooptical studies of two types of novel $\mathrm{CdTe} / \mathrm{CdMgTe}$ quantum well structures having a precisely controlled grading of either the quantum well width or the donor concentration in a direction perpendicular to the growth axis. The presence of two-dimensional electron gas of varying concentration produced by the graded modulation doping was evidenced by observation of negatively charged exciton-electron complexes $\left(\mathrm{X}^{-}\right)$.

PACS numbers: $73.20 . \mathrm{Dx}, 78.55 . \mathrm{Et}$

Formation of trions - i.e., negatively (or positively) charged exciton-electron (exciton-hole) complexes $\mathrm{X}^{-}\left(\mathrm{X}^{+}\right)$- and quenching of the excitons by increasing the concentration of two-dimensional electron (hole) gas in the vicinity of the metal-insulator transition has attracted recently a considerable attention. The existence of trions have been hypothesized long ago by Lampert [1] but their observation in bulk semiconductors was hampered by a very small value of the binding energy of the second electron to the exciton (equal to $0.03 \mathrm{Ry}_{\mathrm{ex}}^{*}$ if electron to hole effective mass ratio $m_{\mathrm{e}}{ }^{*} / m_{\mathrm{h}}^{*}=0.5$ ). In low-dimensional structures the binding energy increases considerably. In strictly two-dimensional case the increase is by a factor of 10 [2]. For this reason $\mathrm{X}^{-}$complexes were in fact observed experimentally for the first time in $\mathrm{CdTe} / \mathrm{CdZnTe}$ quantum wells (QWs) [3] and, later, in

*This work is supported in part by the Committee for Scientific Research (Poland) through grant 8T11B 01411 and by Volkswagen Foundation (Germany). 
structures involving III-V compounds [4]. So far, the method employed for precise studies of an evolution of optical properties of low-dimensional modulation-doped structures with an increase in 2D carrier concentration involved an application of the gate voltage $[4,5]$. In this work we propose an alternative approach which makes use of two types of novel quantum well structures made of CdTe with CdMgTe barriers.

The structures have been grown by a modified molecular beam epitaxy method, described below, on (100)-oriented, epi-ready GaAs substrates with $4.5 \mu \mathrm{m}$ thick CdTe buffer layers deposited prior to growth of the structures in a separate MBE process. The substrates were rectangularly shaped having the length of $50 \mathrm{~mm}$ and the width of $7 \mathrm{~mm}$. Elemental $\mathrm{Cd}, \mathrm{Mg}$ and Te were used as a source material while iodine doping was performed from an effusion cell filled with $\mathrm{ZnI}_{2}$. In order to evaluate their quality, the structures have been extensively studied by magnetooptical methods, including photoluminescence (PL), photoluminescence excitation (PLE) and reflectivity spectroscopies.

In structures of the first type, the concentration of iodine donors was varied in the controlled fashion along the long axis of substrate. To achieve this in our EPI-620 MBE system we used the following routine. First, $0.7 \mu \mathrm{m}$ thick $\mathrm{Cd}_{1-x} \mathrm{Mg}_{x} \mathrm{Te}$ buffer was deposited while rotating the substrate, followed by 15 periods of $20 \AA \mathrm{CdTe} / 20 \AA \mathrm{Cd}_{1-x} \mathrm{Mg}_{x} \mathrm{Te}$ superlattice, $500 \AA \mathrm{Cd}_{1-x} \mathrm{Mg}_{x} \mathrm{Te}$ barrier, $80 \AA \mathrm{CdTe} \mathrm{QW}$ and $100 \AA \mathrm{Cd}_{1-x} \mathrm{Mg}_{x} \mathrm{Te}$ of an undoped spacer. Then, the substrate rotation was arrested and the growth of iodine doped $\mathrm{Cd}_{1-x} \mathrm{Mg}_{x}$ Te region commenced while the main shutter was moved in four $11 \mathrm{~mm}$ wide steps with their edges perpendicular to the long edge of the substrate. This resulted in different thickness of the doped region $(0,10,20$ and $50 \AA$, respectively) and, thus, in different number of introduced donors, in different parts of the structure. After completion of the doping procedure the remaining $500 \AA$ undoped barrier was grown while again rotating the substrate (see the scheme on the right hand side of Fig. 1). Such grow th procedure allowed to produce a modulation-doped structures having two-dimensional electron gas (2DEG) of densities varying from 0 at the one end of the sample to about $5 \times 10^{10} \mathrm{~cm}^{-2}$ at the other. The advantage of such structures is that the "quantum wells with different 2D electron concentrations" are otherwise exactly the same, since they are in fact made of the very same QW, fabricated before the wedge-like doping process.

A good quality of the "wedge-doped" structure and the presence of 2DEG of varying concentration was verified by magnetooptical studies. PL intensity collected from this structure for different positions of the exciting laser spot is presented in Fig. 1. The PL spectrum of the undoped part is dominated by the nearly free exciton recombination, while the PL of the most heavily doped part of the structure is dominated by the recombination of $\mathrm{X}^{-}$complexes (and/or donor bound excitons). Spectra from the intermediately doped regions reveal a gradual evolution of the PL between the two extreme doping concentrations. Figure 2 shows magneto-reflectivity of the two opposite parts of "wedge-doped" structure measured at $1.6 \mathrm{~K}$ in the Faraday configuration for both $\sigma^{+}$and $\sigma^{-}$polarizations. Spectra from the undoped region show a single line due to the formation of the neutral exciton $\mathrm{X}$. The reflectivity spectrum in $\sigma^{+}$polarization of the most heav- 


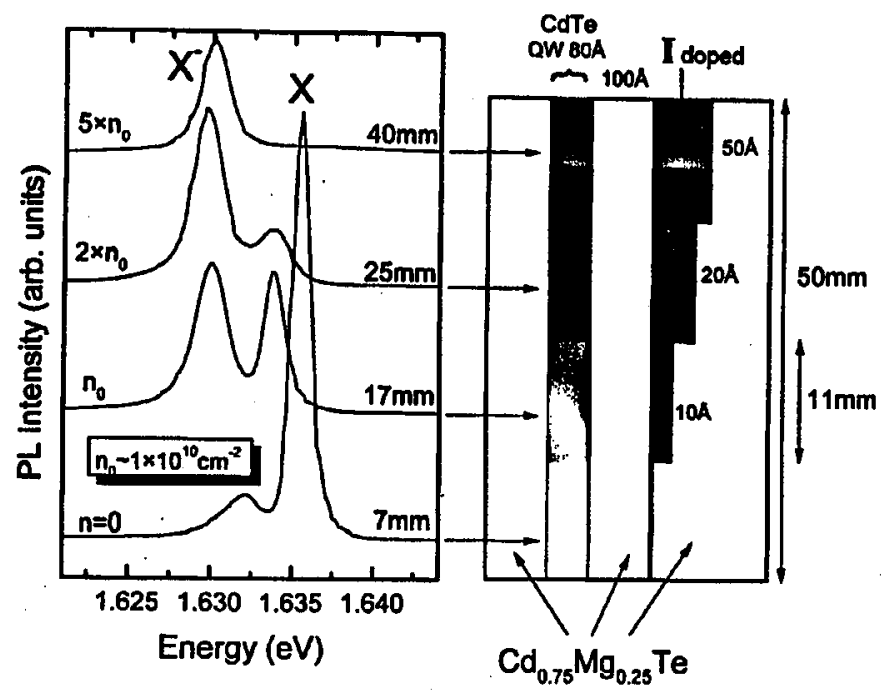

Fig. 1. Photoluminescence intensity of the modulation doped structure with iodine donors introduced $100 \AA$ away from the $80 \AA$ wide $Q W$. Details of the structure design are shown by the scheme on the right hand side. Different shadings of the $\mathrm{QW}$ region represent varying $2 \mathrm{DEG}$ concentration. $T=1.8 \mathrm{~K}, \lambda_{\mathrm{exc}}=496 \mathrm{~nm}$.

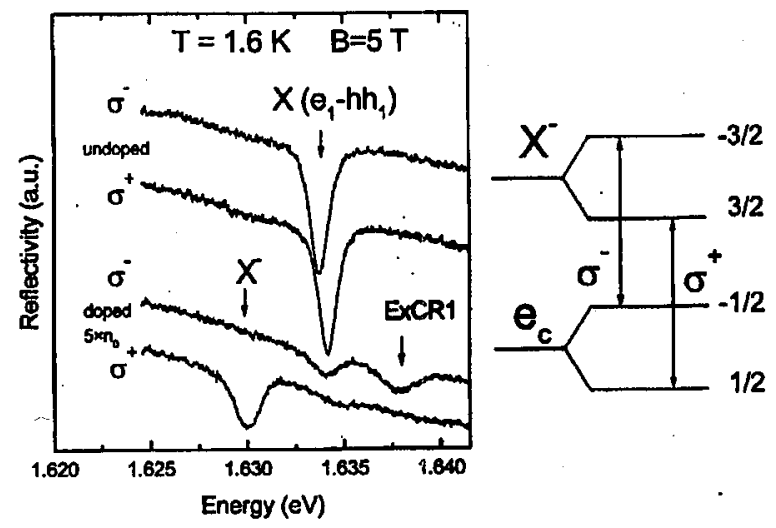

Fig. 2. Reflectivity of two regions (undoped and the most heavily doped) of wedge-doped structure (same as in Fig. 1) measured at $1.6 \mathrm{~K}$ in Faraday configuration using $\sigma^{+}$and $\sigma^{-}$polarized light.

ily doped region is dominated by the line attributed to $\mathrm{X}^{-}$complexes. Strong $\sigma^{+}$ polarization of the $\mathrm{X}^{-}$line is caused by the spin-splitting of the conduction band electrons which leads to a strong preferential occupation of the initial state with spin $+1 / 2$ at low temperatures (see the energy level diagram on the right hand side of Fig. 2) and substantiates the identification of the line [3]. In the $\sigma^{-}$polarization, an additional line due to the photocreation of excitons with simultaneous 
excitation of background electron from lowest Landau level (LL) $(N=0)$ to the next $(N=1)$ LL, marked ExCR, is visible [6].

In the second type of structures, a precisely controlled grading of the $\mathrm{QW}$ width along the long axis of the substrate was achieved. The growth procedure was analogous to that described previously, however, now the main shutter was moved in steps during the growth of the QW region. The resulting "wedge-QW" offers a possibility of tuning the energy levels simply by choosing a proper position on the structure. We succeeded in producing structures containing QWs with widths varying from 30 monolayers (ML) down to $3 \mathrm{ML}$. They emitted bright light with different colors from red to green in one structure, depending on the position of the exciting beam spot. By properly modifying the screen of the flux monitor of our EPI-620 MBE system, so that it works now as a main shutter moving linearly at the distance of about $7 \mathrm{~mm}$ from the substrate surface, we were also able to obtain the boundaries between regions with $\mathrm{QW}$ of different width much sharper (less than $2 \mathrm{~mm}$ ) than before [7]. The modulation doped "wedge-QW" structure produced by the method described above can be very useful in the planned studies of the well width dependence of the binding energy of the second electron in $\mathrm{X}^{-}$ complexes, the problem not studied in the full range of well width yet [8].

\section{References}

[1] M.A. Lampert, Phys. Rev. Lett. 1, 450 (1958).

[2] B. Stébé, A. Ainane, Superlattices Microstruct. 5, 545 (1989).

[3] K. Kheng, R.T. Cox, Y. Merle d'Aubigné, F. Bassani, K. Saminadayar, S. Tatarenko, Phys. Rev. Lett. 71, 1752 (1993).

[4] A.J. Shields, M. Papper, D.A. Ritchie, M.Y. Simmons, G.A.C. Jones, Phys. Rev. B 51, 18049 (1995).

[5] G. Finkelstein, H. Shtrikman, I. Bar-Joseph, Phys. Rev. B 53, R1709 (1996).

[6] D.R. Yakovlev, V.P. Kochereshko, R.A. Suris, W. Ossau, A. Waag, G. Landwehr, P.C.M. Chrisianen, J.C. Maan, in: Proc. 2Srd Int. Conf. on the Physics of Semiconductors, Berlin 1996, Ed. M. Scheffler, R. Zimmermann, World Scientific, Singapore 1996, p. 2071.

[7] T. Wojtowicz, G. Karczewski, J. Kossut, Acta Phys. Pol. A 88, 631 (1995).

[8] J.L. Osborne, A.J. Shields, M. Pepper, F.M. Bolton, D.A. Ritchie, Phys. Rev. B 53, 13002 (1996). 